DOI: $10.23857 /$ pc.v2i11.386

Recepción: 20 / 06/ 2017

Aceptación: 25/ 07 / 2017

Publicación: 30 / 11 / 2017
Ciencias de la educación

Artículo de investigación

\title{
La cultura inclusiva en el contexto formativo universitario: modelo para su implementación
}

Inclusive culture in the university training context: model for its implementation

A cultura inclusiva no contexto formativo universitário: modelo para implementação

Iliana M. Fernández-Fernández ${ }^{\mathrm{I}}$ iliana.fernandez@uleam.edu.ec

Agustín de la Herrán-Gascón II Agustinherran.12@yahoo.com

Correspondencia: iliana.fernandez@uleam.edu.ec

\footnotetext{
I Doctora en Ciencias Pedagógicas, Maestría en Artes en Educación, Maestría en Educación Especial, Licenciada en Educación Especial, Universidad Laica Eloy Alfaro de Manabí, Manta, Ecuador.

II Docente de la Universidad Autónoma de Madrid, Cantoblanco, Madrid, España.
} 


\section{Resumen}

En este trabajo se presenta un modelo procedimental, para el fomento de la cultura inclusiva en el contexto formativo universitario ecuatoriano, específicamente en la provincia de Manabí. Para ello se realiza un estudio teórico conceptual sobre la inclusión educativa en la universidad, un análisis crítico de la concepción que se maneja en el país y los modelos existentes en otras universidades de América Latina. Se emplean los métodos teóricos como el estudio de fuentes bibliográficas -en las que se incluyen los documentos normativos- y el análisis y síntesis para valorar los elementos conceptuales y metodológicos, que permiten delimitar los subsistemas, componentes, principales relaciones del modelo y las barreras que existen en la actualidad en el contexto universitario. Se abordan las relaciones teóricas entre los subsistemas 'base conceptual de la cultura inclusiva' y 'base metodológica inclusiva' y sus componentes con vistas al incremento creciente de la cultura inclusiva en los docentes de la universidad.

Palabras clave: inclusión; inclusión educativa; modelos de inclusión educativa; base conceptual; base metodológica.

\section{Abstract}

This paper presents a procedural model for the promotion of inclusive culture in the Ecuadorian university training context, specifically in the province of Manabí. For this, a conceptual theoretical study on the educational inclusion in the university is carried out, a critical analysis of the conception that is handled in the country and the models existing in other universities of Latin America. Theoretical methods such as the study of bibliographic sources - including normative documents - and the analysis and synthesis to assess the conceptual and methodological elements, that allow to delimit the subsystems, components, main relations of the model and the barriers that currently exist in the university context. It addresses the theoretical relations between the subsystems 'conceptual basis of inclusive culture' and 'inclusive methodological basis' and its components with a view to the increasing increase of inclusive culture in university teachers.

Keywords: inclusion; educational inclusion; models of educational inclusion; conceptual basis; methodological basis. 


\section{Resumo}

Em este trabalho, preste um modelo de procedimento, para o fomento da cultura inclusiva no contexto formativo universitário ecuatoriano, especificamente na provincia de Manabí. Para isso, realize um estudo teórico conceitual sobre a inclusão educativa na universidade, um relatório crítico da concepção que se manuseia no país e os modelos existentes em outras universidades da América Latina. Se empregando os métodos teóricos como o estudo de fontes bibliográficas -en as que se incluem os documentos normativos e o processo e síntesis para valorar os elementos conceituais e metodológicos, que permitem delimitar os subsistemas, componentes, principais relações do modelo e as barreras que Existem na atualidade no contexto universitário. Se abordar as relações teóricas entre os subsistemas 'base conceitual da cultura inclusiva' e 'base metodológica inclusiva' e sus componentes com vistas ao crescimento crescente da cultura inclusiva nos docentes da universidade.

Palavras chave: inclusión; inclusão educativa; modelos de inclusão educativa; base conceitual; base metodológica.

\section{Introducción}

En Ecuador, la educación superior se encuentra inmersa en profundos cambios para lograr la transformación de todos los procesos sustantivos universitarios, a partir de lo establecido en la Constitución de la República de Ecuador (Asamblea Nacional de Ecuador, 2008), en el Plan Nacional para el Buen Vivir (Secretaría Nacional de Planificación y Desarrollo, 2008) y la Ley Orgánica de Educación Superior (Asamblea Nacional de Ecuador, 2010) donde los estudiantes deben asumir una posición activa, transformadora, productiva, que se involucre en la tarea, que adopte posiciones reflexivas en su actuación y se respete la diversidad.

Los niveles de crecimiento y desarrollo alcanzados por la educación superior exigen, cada vez más, continuar elevando y perfeccionando la calidad del proceso de enseñanza-aprendizaje en todas las áreas. La inclusión en el contexto universitario en los últimos tiempos, ha pasado de ser un mito, para convertirse en una realidad asumida por los profesionales de este nivel bajo condiciones complejas, situándose como una de las regularidades fundamentales las falencias de los profesores en el orden teórico y práctico. 
Las universidades desde su creación han tenido como misión fundamental el desarrollo científico técnico del mundo, a través de los programas que establezcan la generación de la producción del conocimiento científico. En Ecuador, según Fernández, Véliz y Ruiz (2016, p.8), hoy se aspira a un modelo de universidad que presente una elevada concentración de hombres de ciencia y pensamiento, que hacen de ella una institución social con capacidad de generar nuevos conocimientos y de difundirlo y socializarlo.

Los estudiantes con discapacidad, cuando ingresan a la universidad comienzan una nueva etapa de vida diferente y hasta incierta en algunos casos. Primero porque se han educado bajo un modelo donde en la práctica no están incluidos en un sistema todos los componentes de la formación inclusiva. Y, en segundo lugar, en el país, el proceso formativo de la educación básica también adolece de docentes con la formación requerida en los temas de inclusión. Los estudiantes no son preparados para el tránsito a la educación superior y lo que resulta aún más complejo, pese a que se han tomado medidas para el desarrollo de los exámenes de ingreso, los bachilleres con discapacidad no conocen en profundidad la oferta académica de la educación superior, es decir, la orientación vocacional que se realiza es muy incipiente y no les permite elegir una carrera acorde a su realidad y aspiración individual.

Lo que se ha manifestado es expresión de la contradicción, que existe entre la aspiración profesional de los estudiantes con discapacidad y la solidez de la oferta académica de la universidad desde un enfoque inclusivo. Hoy la universidad debe partir de los requerimientos que demanda un proceso de inclusión, en esta dirección. Como se ha expresado en investigaciones precedentes de los autores Fernández, Véliz y Ruiz (2016), es necesario conocer a fondo por los docentes las necesidades, intereses y motivos de los estudiantes con discapacidad. Por otro lado, los profesores deben ir hacia la búsqueda de métodos, que le permitan a este estudiantado asimilar el conocimiento y potenciar el desarrollo de sus habilidades y capacidades, para que puedan enfrentar los retos que le impone la sociedad como futuros egresados de la educación superior.

Como bien señalan algunos autores (Beyer, 2001), las teorías contemporáneas del aprendizaje plantean que los profesores y las profesoras deben ser personas capaces de enseñar en contextos diversos de aprendizaje. Los autores coinciden con éstos, aunque el análisis de la realidad 
educativa universitaria demuestra lo contrario, ya que las mallas curriculares de la mayoría de las carreras universitarias en Ecuador están estructuradas para los estudiantes regulares que no poseen ningún tipo de discapacidad.

Hoy, en todos los ámbitos educativos, se discute qué se entiende por calidad de la educación con igualdad de oportunidades. Ese concepto puede ocultar discriminaciones si no se toman en cuenta los diferentes puntos de partida del proceso de aprendizaje. Los estudiantes presentan diferentes saberes previos que según lo que afirma Cullen (1992), es necesario conocer ese marco previo para poder desencadenar procesos socio-educativos que promuevan una verdadera igualdad.

Por otro lado, de igual manera se coincide con lo planteado por Cullen (1992), porque cuando un docente en su proceso de formación no logra adquirir todos los conocimientos y competencias que demanda el ejercicio de su profesión, resultará difícil que pueda responder a las necesidades de inclusión.

Cuando se buscan alternativas para lograr mayor calidad en la educación superior, uno de los aspectos que se toman en cuenta es la igualdad de oportunidades, sin embargo, por más que se ha suscrito la calidad como sinónimo de igualdad consideramos que esto no es posible, dadas las propias condiciones del sistema educativo ecuatoriano. La igualdad significaría las mismas posibilidades para todos, entendiendo entonces que la calidad educativa incluye la equidad y todavía en los procesos de ingresos de los estudiantes con discapacidad a la universidad, así como en la propia formación del docente universitario existen sesgos que se convierten en barreras psicológicas, pedagógicas y culturales que no permiten desarrollar un proceso inclusivo.

Por todo ello, para mejorar la calidad de la educación es necesario producir transformaciones significativas, en el sistema educativo y profesionalizar la acción del docente para atender la diversidad, la cual es considerada uno de los pilares que permite alcanzar un desarrollo integral para la sociedad.

En tal sentido, resulta importante buscar alternativas que den respuestas a estas necesidades, por lo que constituye una prioridad lograr que en el contexto educativo de la Educación Superior Ecuatoriana se atienda la diversidad, la cual remite al hecho de que todos los alumnos tienen unas 
necesidades educativas individuales propias y específicas para poder acceder a las experiencias de aprendizaje necesarias para su socialización, cuya satisfacción requiere una atención pedagógica individualizada.

A partir del análisis efectuado, se plantea como objetivo de la investigación: presentar un modelo procedimental para el fomento de la cultura inclusiva en el contexto formativo universitario ecuatoriano, específicamente en Manabí.

\section{Metodología}

Fueron empleados los métodos teóricos, como el estudio de fuentes bibliográficas -en las que se incluyen los documentos normativos- y el análisis y síntesis para valorar los elementos conceptuales y metodológicos que permiten delimitar los subsistemas, componentes, principales relaciones del modelo y las barreras que existen en la actualidad en el contexto universitario.

El desarrollo del proceso de modelación para la definición de los componentes del modelo procedimental, con vistas a fomentar la cultura pedagógica inclusiva en el docente universitario, que favorezca la educación de las personas con discapacidad se sustentó en los resultados del análisis crítico-valorativo de las fuentes bibliográficas, la experiencia derivada en el devenir de la investigación (Alegre, 2010; UNESCO, 2009; UTM, 2015; I. Fernández, 2016) y de la reflexión en torno a las principales regularidades obtenidas a través del diagnóstico realizado, en el contexto del proceso formativo de los estudiantes con discapacidad en la Universidad Técnica de Manabí.

\section{Desarrollo}

Un modelo representa las características y relaciones de los componentes del objeto, proporciona explicaciones y sirve de guía para generar hipótesis, a la vez que ofrece explicaciones de carácter metodológico para su uso en la práctica educativa.

Durante el desarrollo de la modelación, se realizó un análisis en cuanto a los rasgos característicos de los modelos pedagógicos que aparecen en la literatura científica, entre los que se pueden señalar: 
- Correspondencia objetiva con el objeto modelado.

- La anticipación.

- Carácter sistémico.

- Que sea corroborable y concretable a diferentes niveles en correspondencia con los procesos que modela.

El modelo se estructura en dos subsistemas, que guardan relaciones de dependencia y subordinación entre cada uno de ellos.

El primer subsistema, denominado "base conceptual de la cultura inclusiva", busca fundamentar conceptualmente aquellos componentes que deben considerarse como parte del conocimiento teórico que un docente universitario necesita saber, comprender y a su vez empoderarse para poder asumir un proceso de inclusión. Se convierten además en la génesis de este proceso. Desde esta concepción, se revelan dos componentes fundamentales: el incentivo pedagógico inclusivo y la comunicación pedagógica inclusiva.

El subsistema "base conceptual de la cultura inclusiva" se define como un proceso dinámico, consciente e integrador que busca el empoderamiento por parte del docente universitario de las actitudes, estilos comunicativos y conciencia de la inclusión en el contexto universitario. Se parte del criterio de que la inclusión y la forma de responder ante los procesos inclusivos la llevamos todos dentro.

La persona es diversa como sujeto y depende de cada cultura, el hecho de entender el fenómeno de la inclusión. Una universidad inclusiva es un nuevo paradigma con respecto a la diversidad. Hasta el presente hemos ido tratando a los estudiantes con discapacidad como responsables de su propia discapacidad. Cuando se habla de inclusión educativa en la universidad, es ella propiamente quien debe buscar alternativas para las respuestas. Trabajar desde la perspectiva de una universidad inclusiva, es trabajar en una universidad para todos los sujetos en un contexto de enriquecimiento personal.

Uno de los componentes fundamentales en el orden teórico con vistas a lograr sentar las bases de una universidad inclusiva lo constituye 'el incentivo pedagógico inclusivo', el cual se define por 
los autores de este trabajo como el proceso de concientización, que tiene lugar en el personal docente universitario para dirigir el proceso formativo de estudiantes con discapacidad, donde hagan parte de su accionar pedagógico todas las acciones que permitan estructurar un enfoque curricular inclusivo sin diferencias.

Las aspiraciones que interactúan en el proceso formativo de estudiantes con necesidades educativas, ya sean de orden social o particular, requieren concientizar el incentivo pedagógico inclusivo para alcanzar la cultura inclusiva en los cuerpos docentes. Conformar expectativas profesionales sobre el desarrollo de ésta, exige que se realicen una serie de valoraciones que dependen, en primer lugar, de considerar el proceso formativo del estudiantado con necesidades especiales como un proceso comunicativo y pertinente para su actuar profesional.

Lo que se plantea anteriormente permite considerar entonces la unidad que se establece entre lo individual y lo social, que se evidencia mediante las aspiraciones-exigencias de la sociedad inclusiva en armonía con las aspiraciones-exigencias formativas de estudiantes con discapacidad. Ante las problemáticas suscitadas a partir de los grandes fenómenos que se dan en un proceso de inclusión en la universidad, dentro de una sociedad que no tiene cultura de ello, las exigencias sociales adquieren matices más complejos. En consecuencia, se requiere de un personal docente universitario competente, donde las aspiraciones y exigencias sociales se concreten en la formación integral del estudiantado, independientemente de cuál sea su necesidad educativa. El incentivo pedagógico inclusivo del personal docente se despliega en torno a un ejercicio profesional consciente, lo que implica el conocimiento y aplicación de métodos y medios de enseñanza que conlleven a la apropiación del conocimiento de sus estudiantes, según sus intereses y necesidades.

La diversidad que existe dentro de un grupo supone la importancia de que el educador o educadora conozca a sus estudiantes, para poderles brindar la atención personalizada que requieren y compartir con este grupo el espacio de crecimiento humano, que representa el proceso formativo. En este sentido, su atención debe centrarse en la formación educativa del alumnado como un todo, y mediante la actividad, debe procurar la formación de su personalidad a partir de la identificación de los principales intereses y necesidades de este. La empatía se 
convierte en su principal instrumento, para accionar favorablemente en la realización personal de estudiantes con discapacidad.

Quienes escriben este texto consideran que el sujeto docente es guía, orientador y mediador del proceso formativo estudiantil, el cual facilita su acercamiento al ideal profesional que aspira y demanda la sociedad. Prepararle para el logro de tales objetivos se convierte también en aspiración y exigencia académica, a través del papel que desempeña la universidad y, directamente, el personal docente.

El incentivo pedagógico inclusivo responde a las exigencias sociales contemporáneas, sobre la inclusión y a las exigencias académicas de la educación superior en Ecuador, a favor de profesionales competentes donde las aptitudes comunicativas docentes juegan un rol fundamental para lograrlo.

Como segundo componente del modelo, se fundamenta la actitud comunicativa inclusiva, la cual se define como el proceso de apropiación por parte de los docentes de los elementos conceptuales básicos relacionados con el dominio de las vías y modelos de comunicación de los estudiantes con discapacidad.

Es la transición de una comunicación excluyente a la incorporación de actitudes comunicativas en los docentes que den respuestas a las necesidades de los estudiantes, en los diferentes ambientes de aprendizaje. Se trata de buscar un proceso de comunicación asertivo, donde prevalezca el respeto a la diferencia. Este se relaciona también con los estilos de enseñanza del docente y los métodos, medios y estrategias que emplea durante la clase. De allí que resulta importante definir como otro subsistema de este modelo el espacio metodológico de la clase.

El segundo subsistema se denomina 'base metodológica inclusiva' y se define como el proceso que contribuye a la formación de la personalidad del estudiante con discapacidad, a través de la clase, constituyéndose esta en la vía fundamental mediatizadora para la adquisición por parte de los estudiantes de los conocimientos, procedimientos, normas de comportamiento, valores; es decir, la apropiación de la cultura legada por las generaciones precedentes, la cual hace suya 
como parte de la interacción en los diferentes contextos sociales específicos donde cada estudiante con discapacidad se desarrolla.

El primer componente de este subsistema lo constituye la 'intencionalidad metodológica inclusiva', que se define como un proceso que direcciona los procedimientos metodológicos a seguir por el docente para la conducción del proceso de enseñanza-aprendizaje de los estudiantes con discapacidad en cada clase.

Uno de los aspectos fundamentales que deben ser contemplados como parte de la intencionalidad metodológica con un enfoque inclusivo lo constituye la motivación, el cual es un factor impulsor esencial en la actividad. El logro de ésta deberá constituir un requerimiento importante en la dirección del proceso de enseñanza-aprendizaje de los estudiantes con discapacidad. Por supuesto, no sólo es suficiente lo referido anteriormente para producir el cambio esperado dentro de un proceso de inclusión en las aulas de la educación superior. Se imponen otros requerimientos como la realización del diagnóstico inicial de los estudiantes con discapacidad, el conocimiento profundo de ellos en cuanto a sus necesidades, posibilidades, intereses y motivaciones, tanto en el sentido general respecto a la edad, como en lo específico de los diferentes estudiantes, sus problemas afectivos, su conducta en el grupo, entre otros aspectos, en que tanto el conocimiento con el que actúe el docente para lograr el clima favorable deseado, como su sensibilidad en la apreciación de estos aspectos, le permitirá conducir un proceso inclusivo con la pertinencia que se requiere.

Otro aspecto importante en la organización y dirección del proceso de enseñanza-aprendizaje inclusivo, lo constituyen las formas de la actividad colectiva, que juegan un papel importante como elemento mediatizador para el desarrollo individual de los estudiantes con discapacidad. Las acciones bilaterales y grupales dentro de la clase, ofrecen la posibilidad de que se traslade de un estudiante a otro, o del docente al estudiante, elementos del procedimiento que pueden faltarle (qué) y el procedimiento a seguir en la realización de la tarea (cómo).

Mediante un análisis conjunto, el estudiante con discapacidad puede completar y reajustar sus puntos de vista individual por medio del conocimiento de diferentes criterios y alternativas para 
la solución delas tareas (ayudas), tanto respecto al contenido en si como, al procedimiento de trabajo.

Esta actividad colectiva favorece que el estudiante con discapacidad pueda expresar lo que piensa, y reflexione respecto a las aportaciones que otros estudiantes y el propio docente le pueda ofrecer, dándose apertura de esta forma al desarrollo del trabajo educativo.

Una vez que el docente se va apropiando de todos los aspectos antes descritos y que son importantes desarrollar con el estudiante discapacitado en una clase, pero que además los logra implementar de forma sistemática, es que se puede plantear que va surgiendo la cultura inclusiva en la educación superior.

El segundo componente del modelo se denomina cultura pedagógica inclusiva, el cual trasciende los límites del docente preparado solo para dirigir una buena clase por aquella que exige conocimientos, sobre los cuales el docente puede sustentar su juicio crítico acerca de la realidad (pedagógica, en este caso) y expresar pautas, reglas, actitudes, un soporte cosmovisivo y un sistema de valores que se concreten de manera permanente en el proceso interactivo, en los modos de actuación de profesores y estudiantes con discapacidad y en las funciones esenciales que desempeña la Universidad.

Para que cada docente universitario, logre realizar con eficiencia la labor inclusiva, debe conocer profundamente a cada estudiante, esto significa dominar su nivel de desarrollo y sus potencialidades en toda su dimensión y fuerza, incluyendo sus sentimientos y sus orientaciones valorativas hacia las esferas esenciales de su vida: la universidad, la familia, el estudio, el trabajo, su conducta, así como las esferas de la personalidad en las que se presentan carencias. La cultura pedagógica inclusiva, se constituye en la síntesis del saber hacer de un docente cuando realmente está preparado para atender a los estudiantes con discapacidad.

En la figura 1, se puede apreciar la representación gráfica del modelo.

Relaciones teóricas para fomentar la cultura pedagógica inclusiva en el docente universitario de la educación de las personas con discapacidad. 

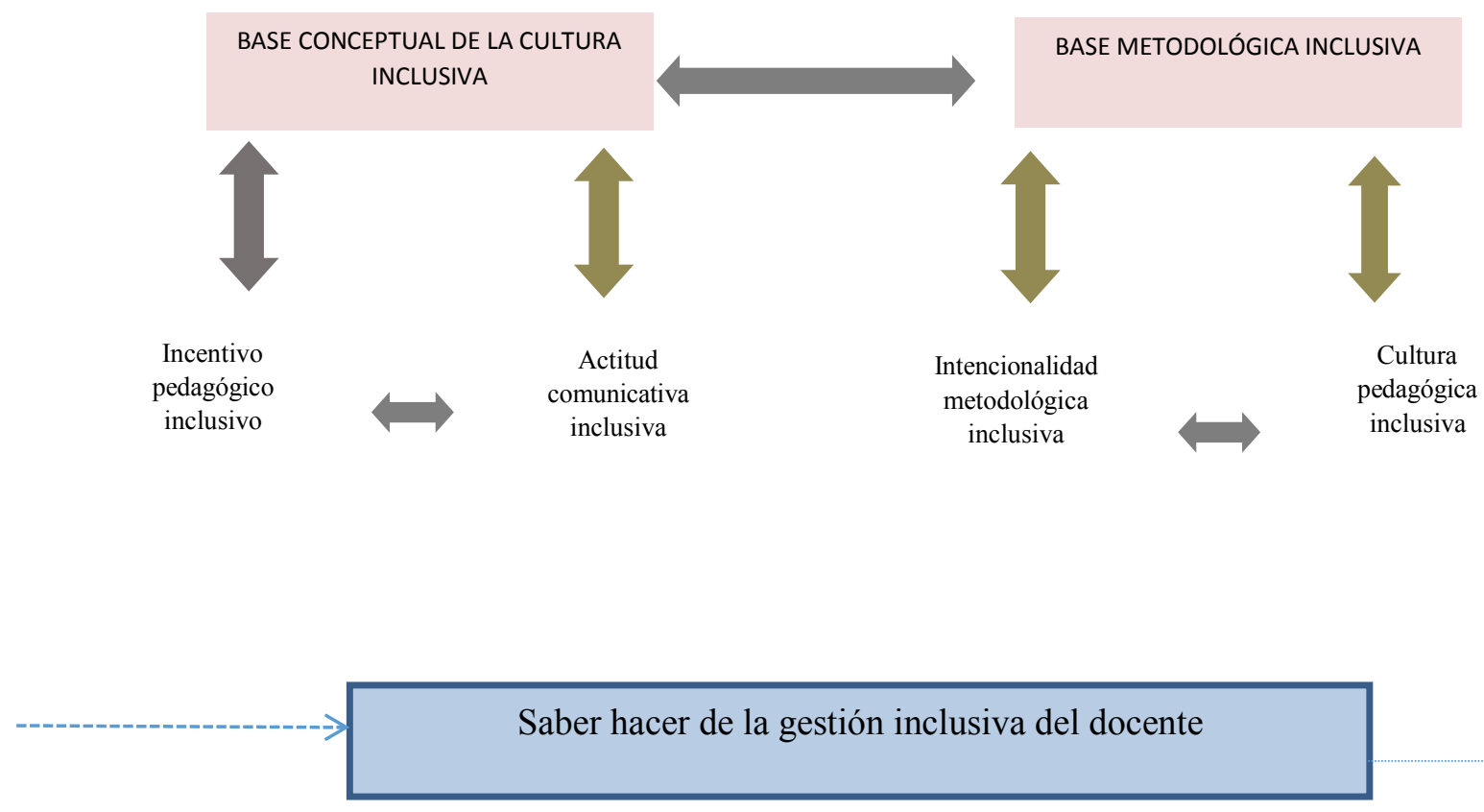

Fig1: Representación gráfica de las relaciones teóricas

Fuente: Elaboración propia

\section{Conclusiones}

Entre los subsistemas y componentes del modelo descrito, se dan un grupo de relaciones esenciales que permiten fundamentar de forma práctica un proceso de inclusión en la universidad, ellas son:

- El modelo fundamentado es expresión del proceso de inclusión de los estudiantes con discapacidad en la universidad, a un nuevo nivel cualitativo de la contradicción entre el carácter específico del proceso de atención de los estudiantes con discapacidad en formación en la universidad y el carácter integral de su desempeño profesional una vez titulados, la cual en su lógica interna impulsa una relación de coordinación entre la base conceptual de la cultura inclusiva y la base metodológica inclusiva. 
- La base conceptual de la cultura inclusiva, adquiere una connotación especial dentro del proceso de inclusión, a través de la dinámica que se da entre el incentivo pedagógico inclusivo y la actitud comunicativa inclusiva.

Este modelo se sintetiza a partir de las relaciones de dependencia y subordinación que se dan entre cada uno de los componentes descritos, donde la unidad dialéctica de ellos trasciende a la sinergia del sistema en el 'saber hacer de la gestión inclusiva del docente' como cualidad esencial que emerge de la modelación efectuada.

\section{Referencias bibliográficas}

Alegre O.M. (2010). Capacidades docentes para atender la diversidad. Una propuesta vinculada a las competencias básicas. Recuperado el enero de 2014, de http://www.ujaen.es/revista/rei/linked/documentos/documentos/11-10.pdf

Asamblea Nacional de Ecuador. (2008). Constitución Política de la República de Ecuador. Ecuador

Asamblea Nacional de Ecuador. (2010). Ley Orgánica de Educación Superior, LOES (2010)

Beyer L.E. (2001). The value of critical perspectives in teacher education. Journal of Teacher Education, http://journals.sagepub.com/doi/abs/10.1177/0022487101052002006

Cullen C. (1992). El papel de la educación en la igualdad de oportunidades foro educativo federal: estrategias para la igualdad de oportunidades de la mujer. Buenos Aires: Consejo Coordinador de Políticas Públicas para la Mujer

Fernández I, M. (2002) Fundamentación pedagógica del proceso de HYPERLINK "http://www.monografias.com/trabajos32/estimulacion-temprana/estimulaciontemprana.shtml" estimulación temprana en los niños con baja visión. ISP "José HYPERLINK "http://www.monografias.com/trabajos28/vigencia-marti/vigenciamarti.shtml" Martí ". Camagüey, Cuba 
Fernández-Fernández, I. M., Véliz-Briones, V. y Ruiz-Cedeño, A. I. (2016). Hacia una cultura pedagógica inclusiva: Experiencias desde la práctica universitaria. Revista Electrónica Educare, 20 (3), 1-15

Secretaría Nacional de Planificación y Desarrollo. (2013). Buen Vivir. Plan Nacional 2013-2017. Todo el mundo mejor. Obtenido de http://documentos.senplades.gob/Plan\%20Nacional\%20Buen\%20Vivir\%2020132017.pdf

UNESCO. (2009). Policy guidelines on inclusion in education [Directrices sobre políticas de inclusión en la educación]. Obtenido de http://unesdoc.unesco.org/images /0017/001778/177849e.pdf

Universidad Técnica de Manabí. (2010). Universidad Técnica de Manabí. Obtenido de http://www.utm.edu.ec/index.asp 